\title{
How serpins transport hormones and regulate their release
}

\author{
Robin W Carrell \\ Randy J Read \\ Cambridge Institute for Medical Research, University of Cambridge, Cambridge, UK.
}

\section{Correspondence:}

Professor Robin Carrell

Trinity College, Trinity Street, Cambridge, CB2 1TQ

United Kingdom

Tel: $\quad+44(0) 1223312970$

Abstract. The adaptation of the serpin framework and its mechanism to perform diverse functions is epitomised in the hormone carriers of the blood. Thyroxine and the corticosteroids are transported bound in a 1:1 ratio on almost identical sites in the two homologous binding-globulins, TBG and CBG. Recent structural findings show an equilibrated, rather than on-and-off, release of the hormones from the carriers, reflecting small reversible movements of the hinge region of the reactive loop that modify the conformational flexibility of the underlying hormone-binding site. Consequently, contrary to previous concepts, the binding affinities of TBG and CBG are not fixed but can be allosterically modified to allow differential hormone delivery. Notably, the two carriers function like protein thermocouples with a surge in hormone release as body temperatures rise in fevers, and conversely a large diminution in free hormone levels at hibernation temperatures. By comparison angiotensinogen, the source of the angiotensin peptides that control blood pressure, does not appear to utilise the serpin mechanism. It has instead evolved a 63 residue terminal extension containing the buried angiotensin cleavage site, which on interaction moves into the active cleft of the renin. The conformational shift involved is critically linked by a labile disulphide bridge. The observation of changes in the redox status of this S-S bridge, in the hypertensive complication of pregnancy, pre-eclampsia, has opened an unexpected level of regulation at what is the initial stage in the control of blood pressure. 


\section{Graphical abstract}
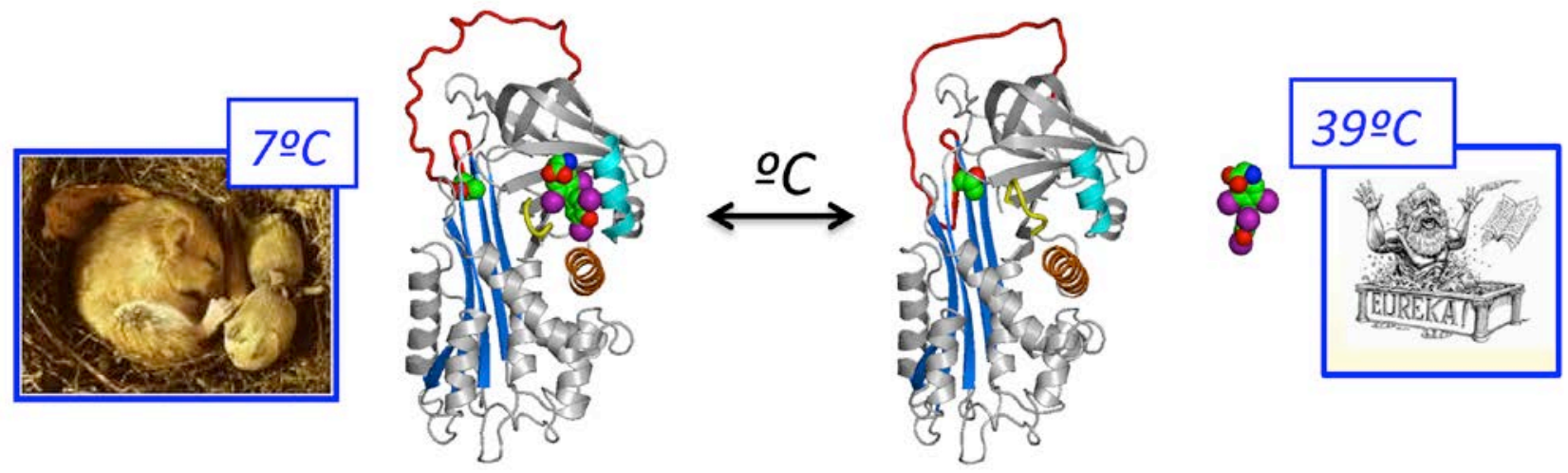

TBG \& thyroxine

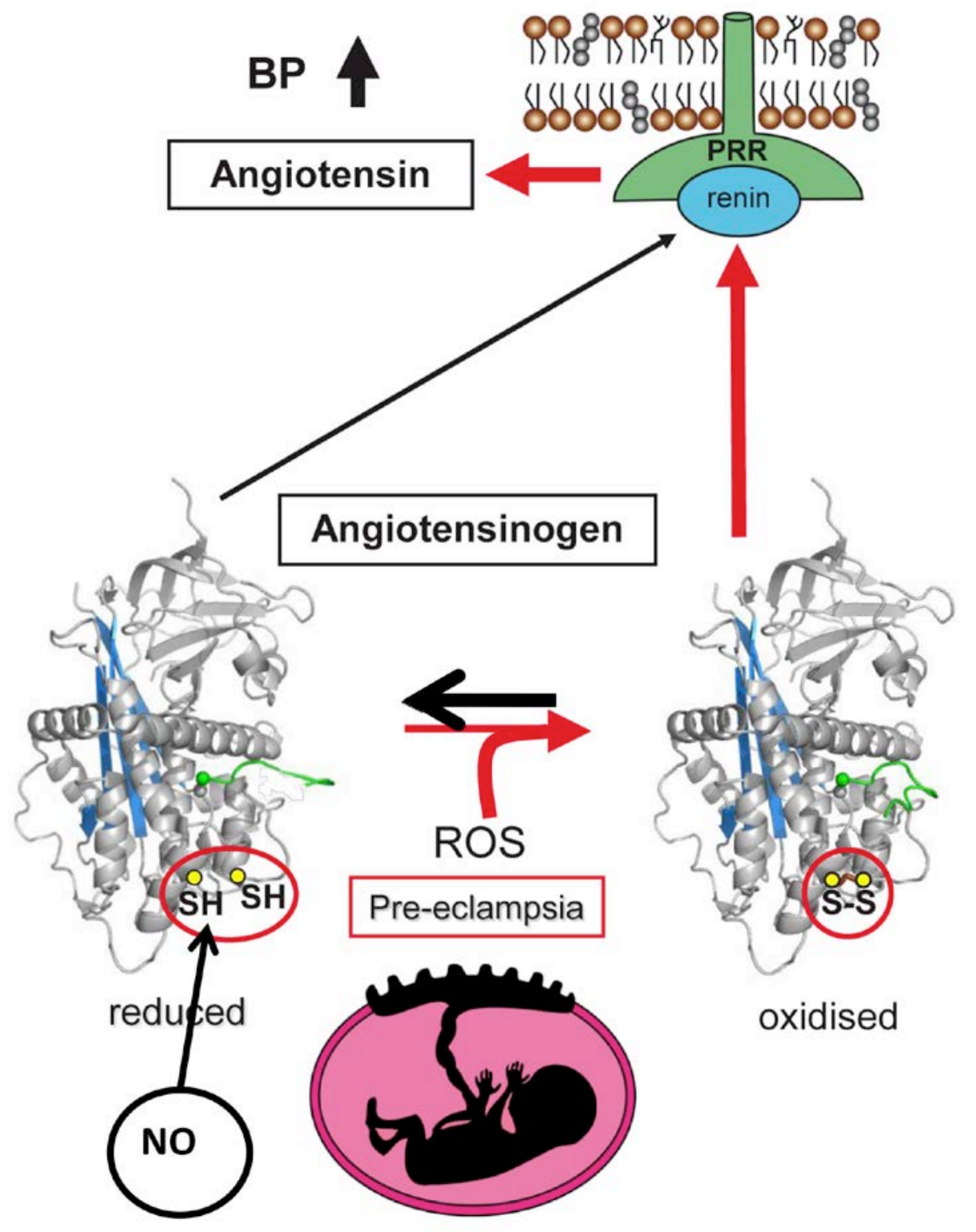

Keywords. Thyroxine, cortisol, TBG, CBG, angiotensinogen, angiotensin, 


\section{Introduction}

The realisation that members of the serpin family all share the same conserved template structure [1] brought with it challenges. A first was how had this single template been adapted to provide such a diversity of functions? But the more puzzling question was why the serpins had been preferentially selected as controlling factors in the critical pathways of higher organisms, including coagulation, fibrinolysis and tissue development and growth? Answers ultimately followed from the structural demonstration of the conformational transition of the serpins that results in the essentially irreversible inactivation of their target proteases [2]. The ability of the serpins to undergo this remarkable change of fold (Fig 1a) had been indicated much earlier in the first of the serpin structures, that of reactive-loop cleaved $\alpha 1$-antitrypsin and then that of an intact serpin, ovalbumin $[3,4]$. The demonstration of the generality of this S-to-R conformational transition from a stressed to a relaxed form in cleaved serpins [5] led to the prediction of its likely adaptation in the intact serpin to allow the modulation of activity [6,7] - a prediction now satisfyingly confirmed in structural detail with the human plasma hormone carriers.

\section{Serpins and the concept of molecular mobility}

It needs to be recalled that the concept of molecular mobility, the ability of proteins to readily undergo major changes in conformation, arose from the early studies of the serpins. It was a controversial concept. At the time of crystallisation of ovalbumin in 1989 there were relatively few solved protein structures and the accepted view amongst crystallographers was that the folds of the known structures were firmly defined, subject to only minor localised changes in conformation. The structure of cleaved $\alpha 1$-antitrypsin led to a necessary, albeit reluctant, acceptance by the field that cleavage of the reactive loop in serpins could induce a radical conformational transition. This was reinforced by the finding of a spontaneous loss of activity in another serpin, PAI-1, due to the irreversible S-to-R incorporation of its intact, uncleaved, reactive loop [8]. Nevertheless, the proposal of the occurrence of a constantly dynamic and fluctuating movement of the reactive loop, as in Fig 1b, was met with scepticism and even, by some, with opposition. There was for years a reluctance to accept that reversible modulatory transitions, as we can now see with the hormonebinding globulins, could occur in the intact serpin. This reluctance had a practical downside as it led to the consideration of serpin function solely in terms of the full S-to-R transition; in terms of simple on-and-off mechanisms or, for the hormone-carrying globulins, a defined transition from a binding to a non-binding state. The revelation that the release of hormones from their serpin transporters in the blood is not an on-and-off process but is subtly modulated by dynamic movements of the intact reactive loop, is now opening new understandings in endocrine medicine $[9,10]$.

\section{Recognition of the hormone carriers}

Initially the recognition and naming of the serpins as a protein family focused on the protease inhibitors in human plasma [11-16]. But at the same time there was also much interest in the identification in human plasma of three non-inhibitory members of the serpin family - the hormone carriers: angiotensinogen [17], thyroxine-binding globulin [18] and corticosteroid-binding globulin [19]. The alignment of the sequences of the three hormone-carriers with other serpins provided immediate clues as to their function. The presence of atypical reactive-centre sequences made it unlikely that these newly identified serpins retained any inhibitory function and this was further excluded with angiotensinogen [20] by a reactive loop hinge sequence (P15-P12) that was incompatible with ready loop-insertion into the A-sheet. TBG and CBG however, having lost any inhibitory activity, do retain the characteristic hinge-sequence of an active serpin. Both were shown to undergo the typical S-to-R serpin transition [21], with proteolytic cleavage of their reactive loops, as in sites of inflammation, resulting in hormone release [21-24]. 
The good sense that this mechanism makes was in a way misleading, as it reinforced the concept that the carriage of thyroxine and corticosteroids is an on-and-off event, with the release from their carriers being triggered by the S-to-R irreversible transition. A hint, however, of a more subtle hormone release mechanism came with the observation by Stein [21] that cleavage of CBG resulted in a partial rather than complete release of the bound cortisol. Although TBG and CBG received much attention in the 1980s, further understandings had to await their crystallisation, which, as with angiotensinogen, proved to be refractory until the new millenium.

\section{Serpins and allosteric modulation}

In the meantime, the wider modulatory potential of the serpin conformational mechanism had become evident, notably with the loop-changes associated with the activation of antithrombin by the heparin pentasaccharide Whereas in the prior structures of antithrombin in the absence of heparin [25] the reactive loop was seen to be partially inserted, a later structure showed that on complexing with heparin an extension of the D-helix took place with an accompanying full expulsion and hence activation of the reactive loop [26]. It was at first assumed that this allosteric activation of antithrombin functioned as an on-off switch but a subsequent structure of the heparinantithrombin complex surprisingly showed, contrary to the previous structure of the same complex, a partial incorporation of the reactive-loop into the central $\beta$-sheet $A$ [27]. The demonstration of these two alternative conformations in the same complex provides direct structural evidence that the intact reactive-loop can move dynamically into and out of the A-sheet, with the binding of heparin resulting in a shift of the equilibrated balance in favour of full exposure of the loop. In this way antithrombin provides a paradigm for the allosteric regulation of serpin activity. It is this in-built potential of the serpins to allow a responsive modulation of activity that explains their selection and adaptation as the controlling factors in key metabolic and intracellular processes, as notably seen with the hormone carrying globulins, TBG and CBG.

\section{Thyroxine and cortisol}

5.1 Thyroxine is the main hormone controlling our activity, with its immediate derivatives regulating cellular oxygen consumption and the metabolism of body and brain. It is a small molecule formed by the linkage of two tyrosines, which are iodinated to give alternative tri- or tetra-iodo forms of the hormone [28]. The steady-state concentration of free thyroxine in the blood is ultimately set centrally by the secretion of the thyroid stimulating hormone TSH, but the maintenance of the concentration of the free hormone, at near 20pM throughout the tissues, results from the equilibrated release of thyroxine from TBG. The binding affinity of TBG for thyroxine is exceptionally tight (Kd 80pM) with TBG acting as a circulating store of the hormone and only $0.03 \%$ of the total thyroxine being in the free form in blood [29]. The binding capacity of circulating TBG is only partially saturated, and it is the percentage saturation of TBG, of $20 \%$ or more, that by the law of mass action determines the concentration of free thyroxine in the blood. This then, till recently, has been the accepted role of both TBG and CBG, as simple carriers that give an equilibrated release to maintain uniform free concentrations of their hormone throughout the tissues. This relationship between the percentage saturation of the binding transport proteins, TBG and CBG, and the free-hormone concentration, is based however on the assumption that the hormone-binding affinity remains a constant throughout. A singular achievement of the structural advances of the last decade has been to overturn this assumption and to reveal an allosteric mechanism in both TBG and CBG that adjusts the affinity of binding to give a responsive release of the carried hormone [30-33]. 
5.2 Cortisol is a steroid hormone critical for the maintenance of life and for survival in stress, which acts by increasing glucose production and suppressing the immune response. Cortisol is transported in a 1:1 ratio by CBG, which serves as a circulating store binding nearly $90 \%$ of the total in the blood. Critically it is the equilibrated release of cortisol from CBG that buffers and regulates the plasma concentration of free cortisol, as analogously so with thyroxine and TBG. The affinity of binding of cortisol by CBG however is much less than that of thyroxine by TBG, with the Kd of CBG being in the nanomolar range versus the picomolar range of TBG. Also, whereas TBG circulates in a partially saturated state, the release of cortisol from the adrenal cortex can exceed the binding capacity of CBG resulting in its complete saturation and hence disproportionately high levels of free cortisol [34,35]. For the most part though, a steady state concentration of cortisol throughout the tissues is maintained with the binding capacity of CBG being only partially saturated. For example in a healthy adult a typical free cortisol concentration of $13 \mathrm{nM}$ [36] will result from a 29\% saturation of CBG. This then, as with TBG and thyroxine, has been the accepted role of CBG as a simple carrier that gives an equilibrated release of the hormone to maintain uniform free cortisol concentrations throughout the tissues. The assumption, as had been so with TBG, was that the hormone-binding affinity of CBG remained a constant throughout.

\section{TBG and CBG}

TBG and CBG are both glycoproteins that retain the typical template serpin structure but with notable differences from the consensus in the sequences of their reactive centre loops. The unique presence in the TBG of all species of a proline at P8 (8-residues prior to the reactive centre - fig 1b) indicates a purposeful impedance to full loop insertion, and the conservation of a proline at P1' is in keeping with TBG's non-inhibitory status. A feature of the reactive loop in CBG, as opposed to TBG and the serpin consensus, is the presence of a valine rather than the polar threonine at the P14 hinge of the reactive loop. The entry of the P14 side-chain into the A-sheet is now believed to trigger the allosteric mechanism (fig 2), the presence of the bulky non-polar valine being in keeping with the more ready and greater magnitude of the allosteric response in CBG versus TBG.

6.1 Structures and the allosteric mechanism of hormone release. The allosteric mechanism and its modulatory potential has recently become evident with the solving of a series of structures of TBG and CBG [30-33, 37]. The two binding globulins have closely similar structures, with thyroxine and cortisol each binding to near identical pockets on the serpin framework, between helices $\mathrm{H}$ and $\mathrm{A}$ and strands 3-5 of the B-sheet (Figs 1a, 2). Each hormone forms a series of hydrophobic and polar bonds with the residues lining the pockets. In TBG the binding of thyroxine is stabilised by a cation- $\pi$ interaction with the positively-charged guanidinium of an arginine, whereas in CBG the main interaction is a $\pi-\pi$ stacking between a homologous tryptophan and the cycloalkene rings of cortisol.

Although the first crystal structures of the TBG and CBG hormone-complexes were interpreted in terms of a tight specificity of binding, subsequent structures emphasise the plasticity of the pockets, with the interactions of the liganded hormones within the pocket being reflected in the affinity rather than precise specificity of binding. Thus both globulins can still bind their respective hormones even after cleavage and transition to the R-form, although with a substantially reduced affinity [9]. Likewise other structures show that the TBG pocket can readily accommodate a range of thyroxine analogues and unrelated drugs and dyes, though again with a lesser affinity [33]. Remarkably, but perhaps not surprisingly, the substitution of the key tryptophan in the binding pocket of CBG by the homologous arginine of TBG changes the binding specificity of CBG from cortisol to thyroxine: in effect the engineered CBG becomes a TBG [38].

Earlier evidence that movements of the reactive loop influenced the ligand affinity of the bindingglobulins came from the demonstration that a shortening of the reactive loop of TBG resulted in an 
increased affinity for thyroxine and conversely a lengthening of the loop gave a decreased affinity [39]. The explanation for this became apparent with the solving of the structure of the TBGthyroxine complex [30]. This, with ancillary evidence from natural variants of TBG [40] indicated the existence of a flip-flop modulatory mechanism, with the affinity of the binding of thyroxine being governed by a limited movement (P14-P8) of the reactive-loop into and out of the A-sheet, as demonstrably occurs with antithrombin. Similarly, TBG crystallised as does active antithrombin, with its reactive-loop partially incorporated in the A-sheet, to the level of a conserved threonine at P14 (Figs 1b, 2). The complete insertion of the sidechain of the P14 threonine is impeded by a similarly conserved underlying tyrosine residue, Tyr 241 in the s2B/3B loop, which critically interacts with underlying peptide loops of the B-sheet that bind to and flank thyroxine in the binding pocket (Fig 2b,c).

Although the displacements resulting from the complete insertion of the side-chain of the P14 Thr can trigger further hormone release, we now believe that the fine-tuning of the physiological balance between the bound and unbound hormones principally occurs with the initial entry of the loop into the A-sheet. This is better described, perhaps, as a nudge-nudge rather than flip-flop movement. The significance of this small initial movement of the loop into and out of the sheet, at the level of P14, became apparent with the determination of the structure of CBG [28]. This showed, as compared to the TBG structure, a fully exposed reactive loop and an accompanying unwinding of the D helix, with the two structures, of TBG and CBG, providing a strikingly comparable identity with the antithrombin heparin-bound and unbound structures, respectively. Subsequent structural studies have confirmed the role of this limited movement of the loop, in TBG as well as CBG, in modifying the affinity of hormone binding. Entry of the reactive-loop will result in an expansion of sheet A with a consequent reordering of the loop that connects strand 2 of the Asheet with the top of the $\mathrm{D}$ helix. This connecting loop is in turn in close contact with loop s2/3B and it in turn with loop s4B/5B that flanks the binding pocket. As in Fig 2b, any slight changes in the packing of these loops will affect the plasticity of the pocket and perturb the polar and hydrophobic bonds that anchor the hormone in the binding site.

Thus contrary to earlier concepts of a simple on-off mechanism, it is now evident that the binding and release of hormones from the carrier-globulins is a much more subtle process, primarily reflecting an allosteric and dynamic equilibrium in both CBG and TBG between high and low affinity conformers of the intact circulating proteins. Inherent to this, the uptake and release of the hormones will be dependent on tissue concentrations of the unbound hormones, with the carrier proteins acting as buffers to evenly maintain the optimal concentrations of the free hormones.

\section{Hormone-binding globulins: physiological and medical implications.}

The revelations, from recent structural advances, of an allosteric release mechanism in both TBG and CBG have direct medical and physiological implications [10].

7.1 Inflammation and cleavage. The finding that the cleavage of TBG and CBG by neutrophil proteases resulted in a loss of binding-affinity initially focused attention on the release of hormones at foci of inflammation. But a realisation of the wider significance of this proteolytic transition has become apparent from a study that defined the range of changes in binding-affinity, Kd, that can take place in plasma CBG [9]. Cleavage of the reactive loop in CBG results in a large diminution but not a loss of binding affinity. There is a tenfold increase in Kd from 32nM to 292nM, which will result in the release of most of the bound cortisol but by no means all, with the retention by the cleaved CBG of a binding saturation near $4 \%$. In a compartmented area where blood flow is sluggish, as in an indurated site of inflammation, a total cleavage of CBG would result in a 4-fold spike in free cortisol, accompanied by a re-equilibration within the compartment of the percentage saturation of the cleaved CBG to $16 \%$, a level at which it could effectively buffer the raised free 
cortisol level. The concept that the cleaved and apparently inactivated CBG might have a role systemically, as a backup buffer to that of the intact circulating intact CBG, has become a likelihood with the recent development of an immunoassay capable of differentiating the two forms of CBG $(41,42)$. The half-life of cleaved CBG in the plasma has now been shown to be the same as that of intact CBG, with in severe sepsis the rise in plasma levels of the cleaved form reaching near equivalence to that of the intact form $[43,44]$. Thus, the structurally-derived finding, that the cleaved hormone carriers retain a significant binding-affinity, has led to the realisation that in severe systemic inflammation there will be two major buffering systems: that of the intact carrier with a high hormone affinity and that of the cleaved carrier with a manifold lower affinity.

7.2 A Protein Thermocouple. Individual proteins have evolved to function optimally at the kinetic flexibility present at $37^{\circ} \mathrm{C}$. This is particularly true of the interactions of proteins with ligands, and it was predictable that the hormone-binding affinity of TBG and CBG would decrease with rises in body temperature [45]. Earlier findings with another serpin, antithrombin, had highlighted a special additional factor, in the temperature dependence of its modulatory mechanism. As with TBG and CBG, the mechanism that controls the activity of antithrombin involves small 'nudge-nudge' movements of the reactive centre loop into and out of the body of the molecule. The sensitivity of these movements to changes in temperature became apparent in patients with mutations in their antithrombin that marginally facilitated the entry of the reactive loop [46]. The affected individuals remain fit and well unless they develop a fever. Then even a small increase in their body temperature, as can occur with a minor infection, is sufficient to increase reactive loop mobility with, in the special case of these mutants, a consequent inactivation of the mutant antithrombin and the consequent onset of thrombosis. Thus an immediate deduction that arose with the solving of the structure of TBG was that its loop-responsive hormone release mechanism would be similarly temperature responsive [30]. As shown in Fig 3, TBG effectively functions as a protein thermocouple: as body temperature decreases and the basal metabolic rate declines, there will be an accompanying increase in binding affinity and an appropriately decreased release of thyroxine. Conversely, when the temperature rises above $37^{\circ} \mathrm{C}$, as in fevers, there will be a boosted release of thyroxine, with the equilibrated concentration of free thyroxine at $39^{\circ} \mathrm{C}$ predictably increasing to near thyrotoxic levels.

Evidence that this potential boost of thyroxine release in fevers is purposeful comes from the presence in an Australian aboriginal population of an adaptive mutation, a replacement of alanine 191 by a threonine, that turns off the temperature-induced acceleration of thyroxine release [29]. Alanine 191 (Fig 2b) is immediately adjacent to the point of entry of the reactive loop into the Asheet of TBG, and its replacement by a polar threonine will predictably affect the H-bond network that links to the bound thyroxine. The replacement critically results (Fig $3 \mathrm{~b}$ ) in an abolition of the accelerated release of thyroxine that otherwise takes place as the temperature rises above $37^{\circ} \mathrm{C}$. Whereas a fever body-temperature of $39^{\circ} \mathrm{C}$ will predictably cause an increase in free thyroxine concentrations to what in health would be thyrotoxic levels, the mutation in the aboriginal TBG limits this increase to within the normal euthyroid range [29]. Deductively, this diminished rise in free thyroxine will be of survival advantage, especially so in infancy, in providing a reduced metabolic response to increased body temperatures in fevers, in an arid environment where ambient temperatures can rise above 45 degrees centigrade.

The effect of changes in temperature on the much more sensitive release mechanism of CBG is even more striking (Fig 3a). Whereas with TBG there will be a $25 \%$ increase in free thyroxine as body temperature rises above $39^{\circ} \mathrm{C}$, with CBG there will be a threefold or more increase in free cortisol $[9,10,47]$. These are changes that rapidly re-equilibrate as the temperature rises and falls. A patient in fever may have a threefold increase in free cortisol but this will revert almost immediately to a normal level as the body is cooled to $37^{\circ} \mathrm{C}$. Similarly, and confusingly so, if a blood sample taken during a fever is then analysed in the laboratory at $37^{\circ} \mathrm{C}$, the result represents what the free 
cortisol concentration is in the blood sample at $37^{\circ} \mathrm{C}$ and not what it was in vivo at the time of sampling. Disconcertingly, measurements of the free hormone determined at room temperature, as often done, are only of direct relevance if the patient is dead!

7.3 Fevers and hot baths. The recently revealed temperature sensitivity of hormone release from TBG and CBG has profound implications in physiology and medicine $[10,29,47]$. The decrease in thyroxine release with decreases in body temperature will play a central part in the cyclical hypothermia and dormancy of small mammals. The changes in humans, though more subtle, will still affect our everyday lives. The reversible changes in binding affinity will occur locally to extremities as well as systemically to the whole body. Conversely the burst of thyroxine and cortisol release with the rise in body core temperature to $39^{\circ} \mathrm{C}$ in a hot bath or sauna explains the accompanying feeling of well-being and mental stimulus - the euphoria and eureka! The new understandings give a glimpse of how bodily processes move into overdrive in fevers. A rise of just one or two degrees $\mathrm{C}$ will automatically raise the blood free thyroxine to a concentration that in health would move into the hyperthyroid range. With corticosteroids the same increase in temperature will lead to a massive release from the circulating store in CBG, to give a two or threefold increase in plasma free cortisol. Fever is clearly a universal defensive response to infection and inflammation, and it is not difficult to see how such increases in active thyroxine and cortisol will be of benefit to the body in a crisis.

\subsection{Conclusions: CBG \& TBG}

The findings with CBG and TBG highlight the reasons for the selection of serpins as the controlling factors in the critical pathways of higher organisms. The inherent ability to modify the functional activity of serpins, by influencing the dynamic movements of their reactive loop, provides a means of adjusting activity to meet the differing requirements of individual tissues and organs. Examples of this responsive modulation are apparent in fevers and inflammation, and the challenge ahead is to identify the receptors and ligands [48] that will predictably influence the conformational flexibility and hormone affinity of CBG and TBG in specific tissues.

\section{Angiotensinogen}

Angiotensinogen plays a pivotal role in physiology, by serving as the substrate in the first and ratelimiting step of the renin-angiotensin system (RAS) that controls blood pressure by influencing salt retention and vasoconstriction [49,50]. The pathway is initiated by the highly specific release of the decapeptide angiotensin- 1 from the $\mathrm{N}$-terminus of angiotensinogen by the enzyme renin. Although angiotensinogen is secreted from the liver and is present in the plasma in relatively high concentration $(0.8 \mu \mathrm{M})$, it is now believed that its primary action occurs at a tissue and cellular level, with the efficiency of cleavage being greatly increased by the interaction of renin with the membrane (pro)renin receptor or (P)RR [51]. Angiotensinogen has typically been regarded as the passive carrier of angiotensin-1, often illustrated with a figure showing the N-terminal peptide emerging from a featureless blob. In light of the understanding that the hormone-binding globulins exploit the whole conformational repertoire of the inhibitory serpins to exercise subtle control of hormone levels, it was reasonable to expect that similar considerations had led to the evolutionary choice of the serpin framework for angiotensinogen as well. Unlike the hormone-binding globulins, however, angiotensinogen was not expected to undergo the sheet expansion found in inhibitory serpins [20]. Though it has emerged from structural and biochemical studies that angiotensinogen indeed is also not a passive carrier, the mechanism by which it appears to play an active role in modulating blood pressure came as a surprise. 
8.1 The crystallographic determination of the structure of angiotensinogen, which triggered these new insights, was the culmination of 20 years of work, in which the initial crystals of human AGT diffracted too poorly and the structure determination was hampered by the lack of known structures of serpins sharing more than about $20 \%$ sequence identity. In the end, it was solved by the simultaneous use of X-ray data from crystals of human, rat and mouse angiotensinogen, in which the electron density could be improved iteratively by averaging images from different crystals [52]. This process yielded first the structure of rat angiotensinogen, which could be used to solve a structure of mouse angiotensinogen at a resolution of $2.2 \AA$, which in turn finally allowed the structure of human angiotensinogen to be determined at $3.3 \AA$ resolution. The 63-residue Nterminal extension containing the angiotensin peptide forms an ordered superstructure wrapped around the serpin body, bound by two new helices (Fig 4a). Surprisingly, the cleavage site was seen to be buried, packed against the body of the protein and inaccessible to renin.

8.2. Interaction with renin. To understand how the cleavage site becomes exposed, crystals of a complex of human angiotensinogen with an inactive mutant of renin were grown. The complex structure (Fig 4b) revealed that the cleavage site, between residues Leu10 and Val11 of angiotensinogen, moves by about $20 \AA$ to enter the renin active site. At the same time, the CD loop of angiotensinogen has to change conformation to avoid a clash with the surface of renin, and it was intriguing to see that these two conformational changes are coupled by a disulphide bridge between Cys18, near the cleavage site, and Cys138, in the CD loop.

Previous work [53,54] had focused attention on this disulphide, which is conserved among different angiotensinogen sequences. The structures, with their conformational changes, showed that it was well placed to play a role in the function of angiotensinogen, if its state could be varied. Indeed, assays of serum samples from healthy volunteers showed that this disulphide exists as a mixture of $40 \%$ reduced and $60 \%$ oxidized [50], unlike the majority of structural disulphides that are essentially completely oxidized. The redox potential of this disulphide was measured as $-230 \mathrm{mV}$ [52], within the range of -184 to $-255 \mathrm{mV}$ found for known allosteric disulphides [53].

Changing the state of the Cys18-Cys238 disulphide, in turn, can affect the function of angiotensinogen as a substrate for renin. In keeping with current understanding that the critical release of angiotensin takes place focally at a cellular level in renal and other tissues [56], the state of the disulphide only has a significant effect in the presence of the (pro)renin receptor; in this circumstance, the catalytic efficiency for release of angiotensin-1 by renin is four-fold greater for oxidized angiotensinogen than for reduced angiotensinogen [52]. Although this is a relatively small effect, it is similar in size to the effect of a Leu10Phe mutation of angiotensinogen associated with increased risk of pre-eclampsia, which causes a two-fold increase in catalytic efficiency [57].

8.3 Oxidation and pre-eclampsia. The biochemical finding of increased catalytic efficiency correlated with the association found between oxidative stress and hypertension in general [58] or pre-eclampsia in particular $[59,60]$. To determine whether the increased oxidative stress of preeclampsia leads, as predicted, to an increase in the proportion of oxidized angiotensinogen, blood samples from women suffering from pre-eclampsia were compared to those from matched normotensive controls at a similar stage of pregnancy. This showed a statistically-significant increase in oxidized angiotensinogen [52,61]. Together, these results suggest that the lability of the Cys18-Cys138 bridge provides an additional mechanism to modulate the control of blood pressure, and that this lability explains, at least in part, the connection between oxidative stress and preeclampsia.

8.4. Divergent evolution. Although this work showed that angiotensinogen is not, in fact, just a passive carrier of angiotensin-1 but rather plays a more active role through the influence of the labile disulphide on catalytic efficiency, it does not answer the question of why evolution chose the serpin framework for this role. No evidence has emerged yet for any influence of the archetypal serpin conformational changes on the role of angiotensinogen in humans or other mammals. 
However, it is intriguing to note that, in the lamprey, angiotensinogen is an active serpin, acting as an efficient heparin-activated inhibitor of thrombin [62,63]. It thus seems likely that angiotensinogen originated as a bifunctional protein with roles in both coagulation and blood pressure control, but that those functions diverged later in evolution, with the protease inhibitory activity having been lost in mammals.

\section{Overall Conclusions}

The solving of structural mechanisms often open new understandings in physiology and medicine. This is so with the finding from the structures of TBG and CBG of an unexpected allosteric regulation of hormone release at tissue level. The surprise with angiotensinogen was not so much in its having a novel hormone release mechanism unrelated to the serpin change of fold, as in the revelation of an unsuspected regulatory process at the very start of the pathway that controls blood pressure. The findings focused on the oxidation of a labile S-S bond and its contributory role in the pre-eclamptic hypertension of pregnancy [52]. What is still unexplored is the likelihood that other factors will be involved in modulating this initial stage in the angiotensin pathway, including nitrosylation [52,64] of the reduced sulphydryl bridge and the contribution of the (pro)renin receptor at both tissue and cellular level [65]. 


\section{References}

1. Huber, R., \& Carrell, R. W. 1989. Implications of the three-dimensional structure of alpha 1-antitrypsin for structure and function of serpins. Biochemistry, 28, 8951-8966.

2. Huntington, J. A., Read, R. J., \& Carrell, R. W. 2000. Structure of a serpin-protease complex shows inhibition by deformation. Nature, 407, 923-926.

3. Loebermann, H., Tokuoka, R., Deisenhofer, J., Huber, R. 1984. Human $\alpha_{1}$-proteinase inhibitor. Crystal structure analysis of two crystal modifications, molecular model and preliminary analysis of the implications for function. J. Mol. Biol. 177, 531-556.

4. Stein, P. E., Leslie, A. G., Finch, J. T., Turnell, W. G., McLaughlin, P. J., \& Carrell, R. W. 1990. Crystal structure of ovalbumin as a model for the reactive centre of serpins. Nature, 347, 99-102.

5. Carrell, R. W., \& Owen, M. C. 1985. Plakalbumin, alpha1-antitrypsin, antithrombin and the mechanism of inflammatory thrombosis. Nature, 317, 730-732.

6. Stein, P. E., Leslie, A. G., Finch, J. T., \& Carrell, R. W. 1991. Crystal structure of uncleaved ovalbumin at $1.95 \AA \AA$ resolution. $J$ Mol Biol, 221, 941-959.

7. Carrell, R. W., Evans, D. L., \& Stein, P. E. 1991. Mobile reactive centre of serpins and the control of thrombosis. Nature, 353, 576-578.

8. Mottonen, J., Strand, A., Symersky, J., Sweet, R. M., Danley, D., Geoghegan, K. F., Gerard, R. D., Goldsmith, E. J. 1992. Structural basis of latency in plasminogen activator inhibitor1. Nature, 355, 270-273.

9. Chan, W. L., Carrell, R. W., Zhou, A., \& Read, R. J. 2013. How changes in affinity of corticosteroid-binding globulin modulate free cortisol concentration. J Clin Endocrinol Metab, 98, 3315-3322.

10. Henley, D., Lightman, S., Carrell, R. 2016. Cortisol and CBG - getting cortisol to the right place at the right time. Pharmacol Ther., 166, 128-135.

11. Petersen, T. E., Dudek-Wojciechowska, G., Sottrup-Jensen, L., Magnusson, S. (Eds.) 1979. Primary structure of antithrombin III: partial homology with alpha1-antitrypsin. In Physiological Inhibitors of Coagulation and Fibrinolysis (Collen, D., Wiman, B., \& Verstaete, M., Eds). pp 45-54. Elsevier/North Holland, Amsterdam.

12. Carrell, R., Owen, M., Brennan, S., Vaughan, L. 1979. Carboxy terminal fragment of human $\alpha$-1-antitrypsin from hydroxylamine cleavage: homology with antithrombin III. Biochem. Biophys. Res. Commun. 91, 1032-1037.

13. Hunt, L. T.,and Dayhoff, M.O. 1980. A surprising new protein superfamily containing ovalbumin, antithrombin-III and alpha1-proteinase inhibitor. Biochem. Biophys, Res. Commun. 95, 864-871

14. Carrell, R. and Travis J. 1985. Alpha1-antitrypsin and the serpins: Variation and countervariation. Trends in Biochemical Science 10, 20-24

15. Carrell, R. W. and Boswell D.R. 1986. Serpins: the superfamily of serine proteinase inhibitors. In: Proteinase Inhibitors (Eds Barrett A and Salvesen G) Chapter12, pp 403-419, Elsevier Biomedical Press, Amsterdam.

16. Carrell, R. W., Pemberton, P.A., and Boswell D.R. 1987. The serpins: Evolution and adaptation in a family of protease inhibitors. Cold Spring Harbor Symposia on Quantitative Biology. LII 527-535

17. Doolittle, R. F. 1983. Angiotensinogen is related to the antitrypsin-antithrombin-ovalbumin family. Science. 222, 417-9.

18. Flink, I. L., Bailey, T. J., Gustafson, T. A., Markham, B. E., Morkin, E. 1986. Complete amino acid sequence of human thyroxine-binding globulin deduced from cloned DNA: close homology to the serine antiproteases. Proc. Natl. Acad. Sci. USA. 83, 7708-7712.

19. Hammond, G. L., Smith, C. L., Goping, I. S., Underhill, D. A., Harley, M. J., Reventos, J., Musto, N. A., Gunsalus, G. L., \& Bardin, C. W. 1987. Primary structure of human 
corticosteroid binding globulin, deduced from hepatic and pulmonary cDNAs, exhibits homology with serine protease inhibitors. Proc.Natl.Acad.Sci.U.S.A, 84, 5153-5157.

20. Stein, P. E., Tewkesbury, D. A., Carrell, R. W., 1989. Ovalbumin and angiotensinogen lack serpin S-R conformational change. Biochem J. 262, 103-7.

21. Pemberton, P. A., Stein, P. E., Pepys, M. B., Potter, J. M., \& Carrell, R. W. 1988. Hormone binding globulins undergo serpin conformational change in inflammation. Nature, 336, 257258.

22. Hammond, G. L., Smith, C. L., Paterson, N. A., \& Sibbald, W. J. 1990. A role for corticosteroid-binding globulin in delivery of cortisol to activated neutrophils. $J$ Clin Endocrinol Metab, 71, 34-39.

23. Jirasakuldech, B., Schussler, G. C., Yap, M. G., Drew, H., Josephson, A., Michl, J. 2000. A characteristic serpin cleavage product of thyroxine-binding globulin appears in sepsis sera. $\mathrm{J}$ Clin Endocrinol Metab. 85, 3996-9.

24. Suda, S. A., Gettins, P. G., Patston, P. A. 2000. Linkage between the hormone binding site and the reactive center loop of thyroxine binding globulin. Arch Biochem Biophys. 384, 316.

25. Schreuder, H. A., de Boer, B., Dijkema, R., Mulders, J., Theunissen, H. J., Grootenhuis, P. D., Hol, W. G. 1994. The intact and cleaved human antithrombin III complex as a model for serpin-proteinase interactions. Nat Struct Biol. 1, 48-54.

26. Jin, L., Abrahams, J. P., Skinner, R., Petitou, M., Pike, R. N., Carrell, R. W. 1997. The anticoagulant activation of antithrombin by heparin. Proc Natl Acad Sci U S A. 94, 14683-8.

27. Johnson, D. J., Huntington, J. A. 2003. Crystal structure of antithrombin in a heparin-bound intermediate state. Biochemistry. 42, 8712-9.

28. Cheng S. Y.; Leonard J.l.; Davis, P.J. 2010. Molecular aspects of thyroid hormone actions. Endocr. Rev. 31, 139-170

29. Qi, X., Chan, W. L., Read, R. J., Zhou, A., \& Carrell, R. W. 2014. Temperature-responsive release of thyroxine and its environmental adaptation in Australians. Proc Biol Sci, 281, 20132747.

30. Zhou, A., Wei, Z., Read, R. J., \& Carrell, R. W. 2006. Structural mechanism for the carriage and release of thyroxine in the blood. Proc Natl Acad Sci U S A, 103, 13321-13326.

31. Klieber, M. A., Underhill, C., Hammond, G. L., \& Muller, Y. A. 2007. Corticosteroidbinding globulin, a structural basis for steroid transport and proteinase-triggered release. $J$ Biol Chem, 282, 29594-29603.

32. Zhou, A., Wei, Z., Stanley, P. L., Read, R. J., Stein, P. E., \& Carrell, R. W. 2008. The S-to$\mathrm{R}$ transition of corticosteroid-binding globulin and the mechanism of hormone release. $J$ Mol.Biol., 380, 244-251.

33. Qi, X., Loiseau, F., Chan, W. L., Yan, Y., Wei, Z., Milroy, L. G., Myers, R. M., Ley, S. V., Read, R. J., Carrell, R. W., \& Zhou, A. 2011. Allosteric modulation of hormone release from thyroxine and corticosteroid-binding globulins. J Biol Chem, 286, 16163-16173.

34. Henley, D. E., Russell, G. M., Douthwaite, J. A., Wood, S. A., Buchanan, F., Gibson, R., Woltersdorf, W. W., Catterall, J. R., \& Lightman, S. L. 2009. Hypothalamic-pituitaryadrenal axis activation in obstructive sleep apnea: the effect of continuous positive airway pressure therapy. J Clin Endocrinol Metab, 94, 4234-4242.

35. Young, E. A., Abelson, J., \& Lightman, S. L. 2004. Cortisol pulsatility and its role in stress regulation and health. Front Neuroendocrinol., 25, 69-76.

36. Ho, J. T., Al-Musalhi, H., Chapman, M. J., Quach, T., Thomas, P. D., Bagley, C. J., Lewis, J. G., \& Torpy, D. J. 2006. Septic shock and sepsis: a comparison of total and free plasma cortisol levels. J Clin Endocrinol Metab, 91, 105-114.

37. Gardill, B. R., Vogl, M. R., Lin, H. Y., Hammond, G. L., \& Muller, Y. A. 2012. Corticosteroid-binding globulin: structure-function implications from species differences. PLoS One, 7, e52759. 
38. Chan, W. L., Zhou, A., \& Read, R. J. 2014. Towards engineering hormone-binding globulins as drug delivery agents. PLoS One, 9, e113402.

39. Grasberger, H., Golcher, H. M., Fingerhut, A., Janssen, O. E. 2002. Loop variants of the serpin thyroxine-binding globulin: implications for hormone release upon limited proteolysis. Biochem J. 365, 311-6.

40. Bertenshaw, R., Takeda, K., Refetoff, S. 1991. Sequencing of the variant thyroxine-binding globulin (TBG)-Quebec reveals two nucleotide substitutions. Am J Hum Genet. 48, 741-4.

41. Lewis, J. G., \& Elder, P. A. 2011. Corticosteroid-binding globulin reactive centre loop antibodies recognise only the intact natured protein: elastase cleaved and uncleaved CBG may coexist in circulation. J Steroid Biochem Mol Biol, 127, 289-294.

42. Lewis, J. G., \& Elder, P. A. 2013. Intact or "active" corticosteroid-binding globulin (CBG) and total CBG in plasma: determination by parallel ELISAs using monoclonal antibodies. Clin Chim Acta, 416, 26-30.

43. Lewis, J. G., Saunders, K., Dyer, A., \& Elder, P. A. 2015. The half-lives of intact and elastase cleaved human corticosteroid-binding globulin (CBG) are identical in the rabbit. $J$ Steroid Biochem Mol Biol, 149, 53-57.

44. Nenke, M. A., Rankin, W., Chapman, M. J., Stevens, N. E., Diener, K. R., Hayball, J. D., Lewis, J. G., \& Torpy, D. J. 2015. Depletion of high-affinity corticosteroid-binding globulin corresponds to illness severity in sepsis and septic shock; clinical implications. Clin Endocrinol (Oxf), 82, 801-807.

45. Mickelson, K. E., Forsthoefel, J., \& Westphal, U. 1981. Steroid-protein interactions. Human corticosteroid binding globulin: some physicochemical properties and binding specificity. Biochemistry, 20, 6211-6218.

46. Beauchamp, N. J., Pike, R. N., Daly, M., Butler, L., Makris, M., Dafforn, T. R., Zhou, A., Fitton, H. L., Preston, F. E., Peake, I. R., \& Carrell, R. W. 1998. Antithrombins Wibble and Wobble (T85M/K): archetypal conformational diseases with in vivo latent-transition, thrombosis, and heparin activation. Blood, 92, 2696-2706.

47. Cameron, A., Henley, D., Carrell, R., Zhou, A., Clarke, A., \& Lightman, S. 2010. Temperature-responsive release of cortisol from its binding globulin: a protein thermocouple. J Clin Endocrinol Metab, 95, 4689-4695.

48. Caldwell, J. D., Jirikowski G. F. 2014. Sex hormone binding globulin and corticosteroid binding globulin as major effectors of steroid action. Steroids. 81,13-16.

49. Cleland, S. J., Reid, J. L. 1996. The renin-angiotensin system and the heart: a historical review. Heart. 76, 7-12.

50. Dickson, M. E., Sigmund, C. D. 2006. Genetic basis of hypertension: revisiting angiotensinogen. Hypertension. 48, 14-20.

51. Nguyen, G., Delarue, F., Burckle, C., Bouzhir, L., Giller, T., Sraer, J. D. 2002. Pivotal role of the renin/prorenin receptor in angiotensin II production and cellular responses to renin. $\mathrm{J}$ Clin Invest. 109, 1417-27.

52. Zhou, A., Carrell, R. W., Murphy, M. P., Wei, Z., Yan, Y., Stanley, P. L., Stein, P. E., Broughton Pipkin, F., Read, R. J. 2010. A redox switch in angiotensinogen modulates angiotensin release. Nature 468, 108-11.

53. Streatfeild-James, R. M., Williamson, D., Pike, R. N., Tewksbury, D., Carrell, R. W., Coughlin, P. B. 1998. Angiotensinogen cleavage by renin: importance of a structurally constrained N-terminus. FEBS Lett. 436, 267-70.

54. Gimenez-Roqueplo, A. P., Celerier, J., Schmid, G., Corvol, P., Jeunemaitre, X. 1998. Role of cysteine residues in human angiotensinogen. Cys232 is required for angiotensinogen-pro major basic protein complex formation. J Biol Chem. 273, 34480-7.

55. Cook KM and Hogg PJ. 2013. Post-translational control of protein function by disulfide bond cleavage. Antioxidants \& Redox Signaling 18, 1987-2015. 
56. Kobori, H., Nangaku, M., Navar, L. G., Nishiyama, A. 2007. The intrarenal reninangiotensin system: from physiology to the pathobiology of hypertension and kidney disease. Pharmacol Rev. 59, 251-87.

57. Inoue, I., Rohrwasser, A., Helin, C., Jeunemaitre, X., Crain, P., Bohlender, J., Lifton, R. P., Corvol, P., Ward, K., Lalouel, J. M. 1995. A mutation of angiotensinogen in a patient with preeclampsia leads to altered kinetics of the renin-angiotensin system. J Biol Chem. 270, 11430-6.

58. Harrison, D. G., Gongora, M. C. 2009. Oxidative stress and hypertension. Med Clin North Am. 93, 621-35.

59. Burton, G. J., Jauniaux, E. 2004. Placental oxidative stress: from miscarriage to preeclampsia. J Soc Gynecol Investig. 11, 342-52.

60. Myatt, L. 2010. Review: Reactive oxygen and nitrogen species and functional adaptation of the placenta. Placenta. 31 Suppl, S66-9.

61. Rahgozar, S; Amirian, T; Qi, M; Shahshahan, Z; Entezar-E-Ghaem, M; Ghasemi Tehrani, H; Miroliaei, M; Krilis, SA; Giannakopoulos, B. 2015. Improved Assay for Quantifying a Redox Form of Angiotensinogen as a Biomarker for Pre-Eclampsia: A Case-Control Study. PLoS ONE, 2015 vol. 10(8) pp. e013590

62. Wang Y, Köster K, Lummer M and Ragg H. 2014. Origin of serpin-mediated regulation of coagulation and blood pressure. PLoS One 9, e97879.

63. Wei H., Cai H.,Zhang F., Huang X., Feng L., Zhang R., Wang Y., RaggH., Zheng Y., Zhou A. 2016. Heparin binds lamprey angiotensinogen and promotes thrombosis inhibiton through a template mechanism. J Biol Chem 2016 Sep28. Pii: jbc 725895. [Epub ahead of print].

64. Wilcox C. S. 2005. Oxidative stress and nitric oxide deficiency in the kidney: a critical link to hypertension? Am. J. Physiol. Regular. Integr. Comp. Physiol. 289, R913-R935

65. Danser A H. 2015. The Role of the (Pro)renin Receptor in Hypertensive Disease. Am. J. Hypertens., 2015 vol. 28(10) pp. 1187-96

\section{Acknowledgements}

This research was supported by the Wellcome Trust (Principal Research Fellowship to RJR, grant 082961/Z/07/Z) and by the British Heart Foundation grant 12/41/29679. The research was facilitated by a Wellcome Trust Strategic Award (100140) to the Cambridge Institute for Medical Research. 


\section{Figures}

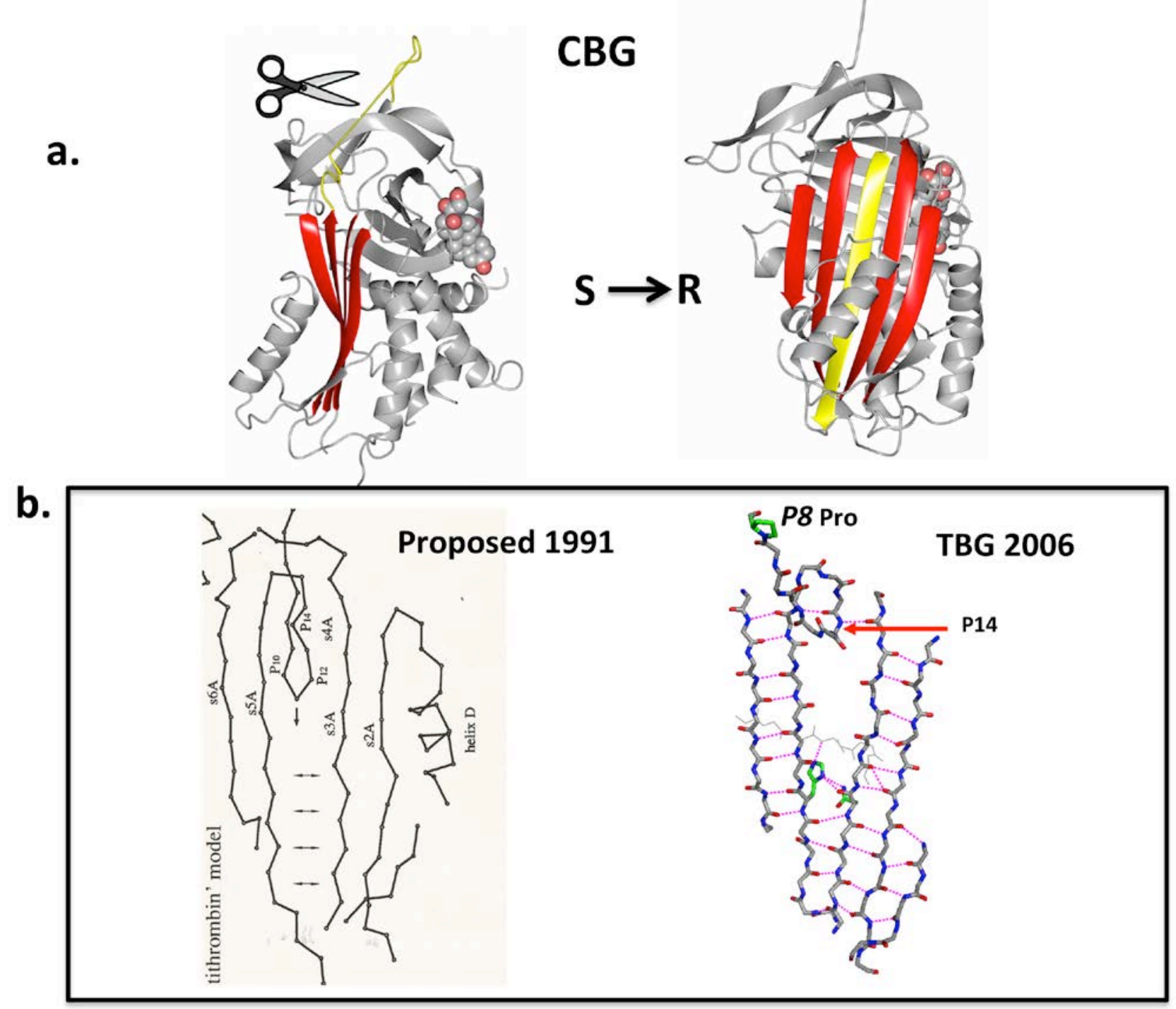

Figure 1. Serpins and mechanisms of hormone release. a. Structures of the CBG-cortisol complex, showing the S-to-R change on cleavage of the reactive loop (yellow) in a side-view of CBG (PDB entry 2v95 [31], on left) and its insertion as the middle strand of the A-sheet (red) in a frontal view on right (PDB 2vdy [32]). Note that cortisol (space-filled) remains bound here to the cleaved-CBG on right. b. The earlier prediction by Stein et al 1991 [6], of reversible movements of the intact reactive loop in-and-out of the A-sheet (PDB 1ova, on left) are borne out in crystallographic detail in TBG on right (PDB 2ceo [30]). The imino-acid proline at P8 will impede full insertion of the loop. 

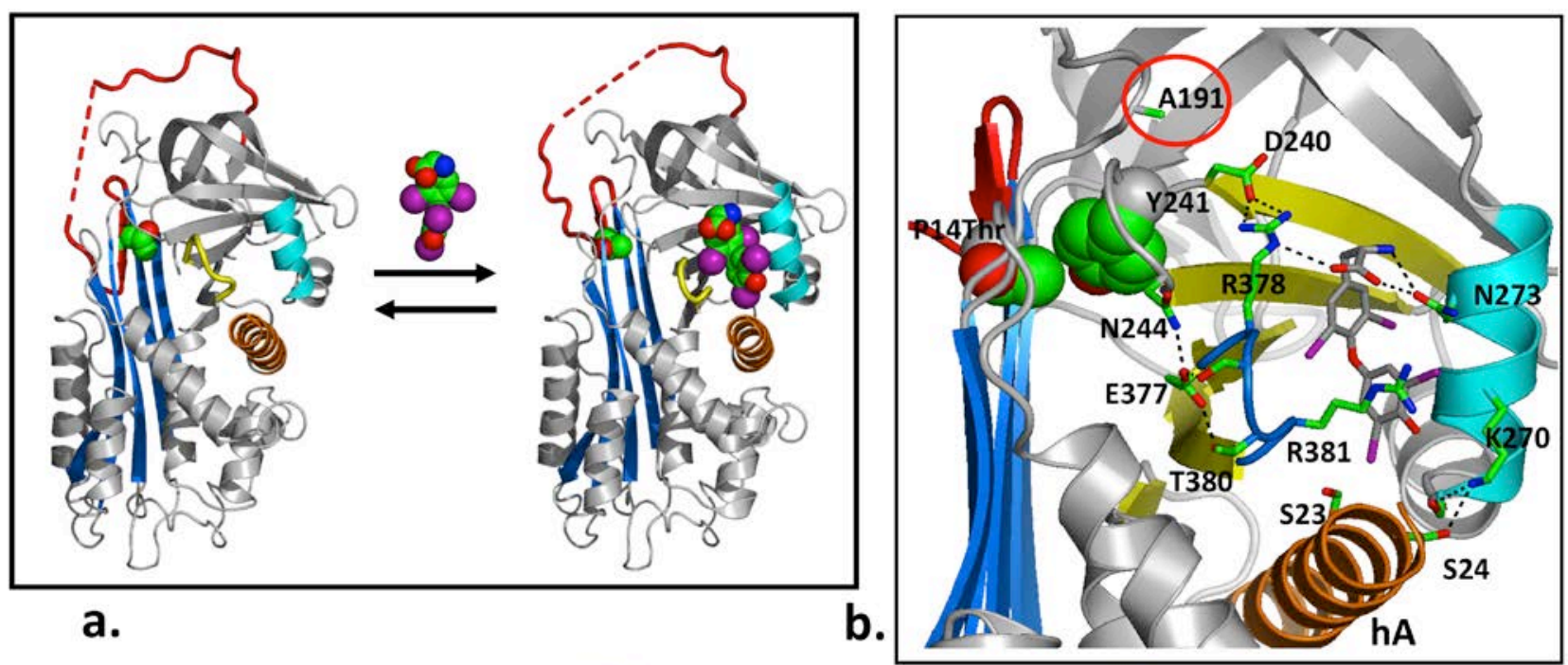

a.

b.

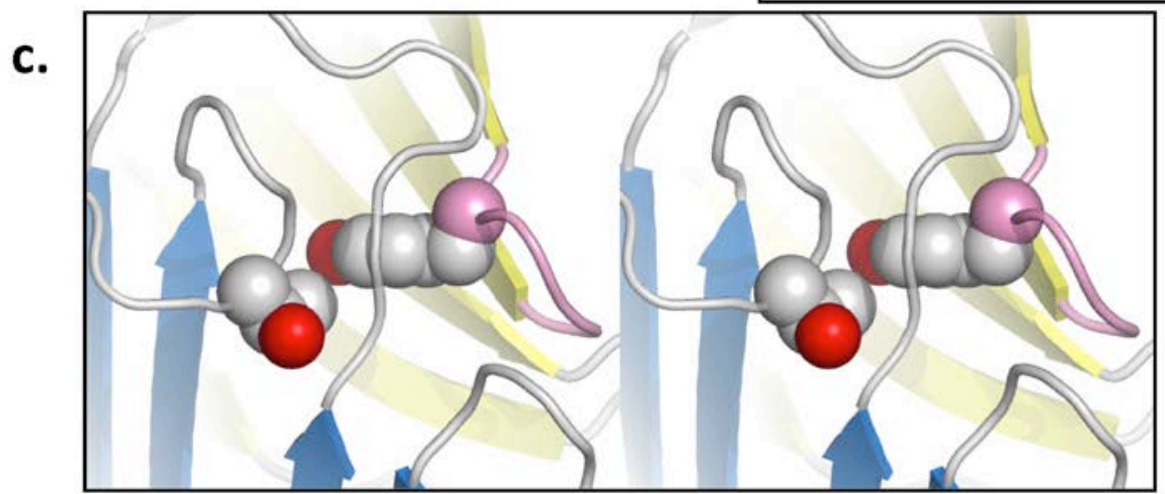

Figure 2. Modulating binding affinity. Hormone pocket of TBG showing interactions with thyroxine [30]. a. Small movements of the reactive loop with insertion of the P14 side chain (space-filling) will affect the plasticity and $\mathbf{b}$. perturb the hydrogen bonding network stabilising the binding of thyroxine (sticks) within the pocket (PDB 2ceo). Circled in red is Ala191 the replacement of which abolishes the 'fever' boost in thyroxine release as in Fig 3. c. Stereo view showing how entry of the P14 side-chain (space-filling left) will sterically displace the underlying Tyr241 (right), as labelled in b. 
a

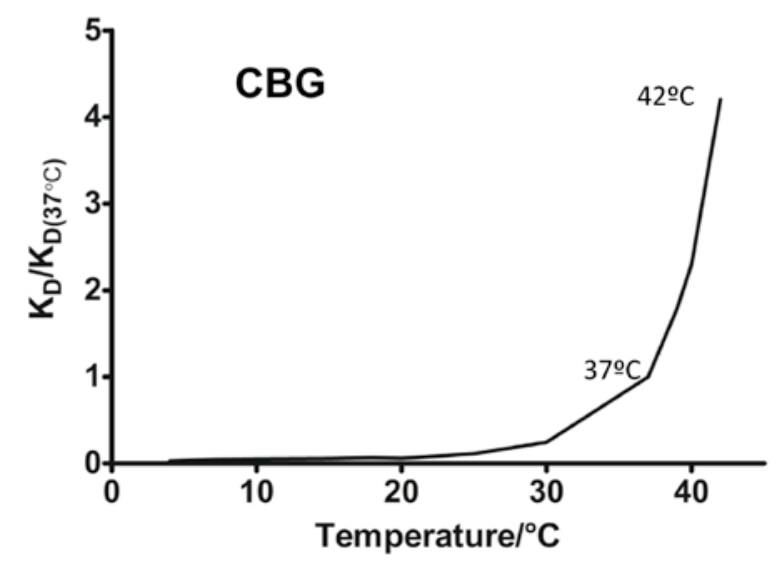

b

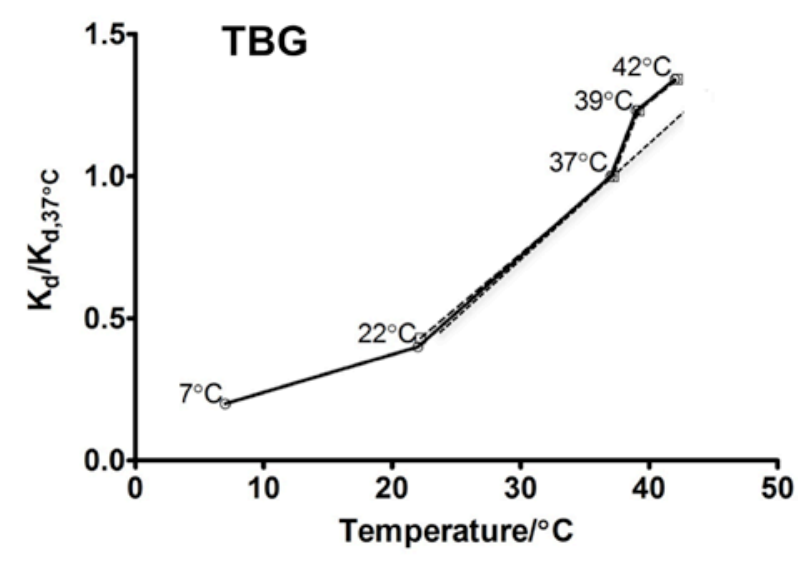

Figure 3. Temperature responsive hormone release. Outline plots of the change in ratios of the binding affinities (Kd/Kd37) with temperature. a. With CBG [9] there will predictably be a near fivefold increase in free cortisol with a rise in body temperature to $42^{\circ} \mathrm{C}$; $\mathbf{b}$. with TBG [29] the increase in released thyroxine will be much less (full line), but its purposeful occurrence is evidenced (dashed line) by a mutation A191T (Fig 2b) in the Australian aboriginal that negates the boosted increase at temperatures above $37^{\circ} \mathrm{C}$. A control is provided by an accompanying mutation in the aboriginal L283F that by itself (heavy dashed line) is superimposable on that of wild-type TBG. 

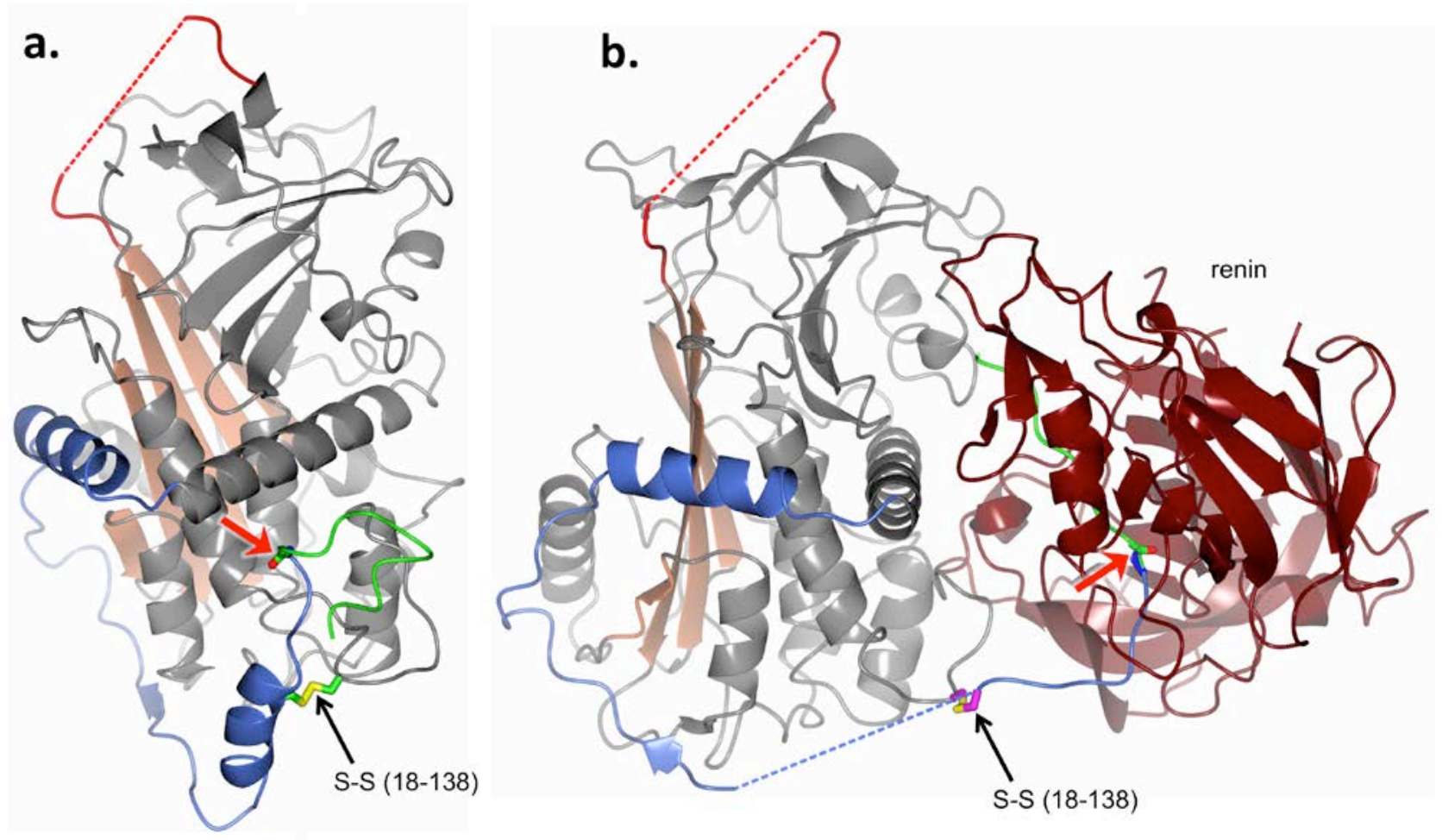

Fig 4. Angiotensinogen and its complex with renin: a. Showing the amino-terminal superstructure anchored to the serpin framework of angiotensinogen (PDB 2wxw [52]) by two helices (dark grey) with the red arrow indicating the buried renin-cleavage site. b. Interaction with renin displaces a protruding CD peptide loop with the concerted movement of the angiotensincontaining terminal into the active-site cleft of renin (PDB 2x0b [52]). 